\title{
Effectiveness of Comprehensive Traditional Chinese Therapy in Treating Granulomatous Lobular Mastitis: a Systematic Review and a Meta-analysis
}

Hongyi Liang

Shandong University of Traditional Chinese Medicine https://orcid.org/0000-0002-3864-6777

Hanhan Chen

Shandong University of Traditional Chinese Medicine Affiliated Hospital

Guangxi Shi

Shandong University of Traditional Chinese Medicine Affiliated Hospital

Xiaofei Liu

Shandong University of Traditional Chinese Medicine Affiliated Hospital

Jingwei Li ( $\nabla$ weilandetian2000@163.com )

Shandong University of Traditional Chinese Medicine Affiliated Hospital https://orcid.org/0000-00015864-7307

\section{Research}

Keywords: Granulomatous lobular mastitis, Comprehensive traditional Chinese therapy, Systematic review, Meta-analysis, Effectiveness, Recurrence, Maximum diameter of the mass

Posted Date: December 30th, 2020

DOl: https://doi.org/10.21203/rs.3.rs-136205/v1

License: (9) This work is licensed under a Creative Commons Attribution 4.0 International License. Read Full License 


\title{
Effectiveness of Comprehensive Traditional Chinese Therapy in Treating Granulomatous Lobular Mastitis: a Systematic Review and a Meta-analysis
}

\section{Authors}

Hongyi Liang ${ }^{1}$, Hanhan Chen ${ }^{2}$, Guangxi Shi ${ }^{2}$, Xiaofei Liư ${ }^{2}$, Jingwei $\mathrm{Li}^{2 *}$

\section{Affiliations}

1.College of Traditional Chinese Medicine, Shandong University of Traditional Chinese Medicine, Jinan 250014, Shandong, China.

2.Department of Breast and Thyroid Surgery, Affiliated Hospital of Shandong University of Traditional Chinese Medicine, Jinan 250014, Shandong, China.

\section{E-mails}

\section{6@qq.com}

weilandetian2000@163.com

\begin{abstract}
Background: With the development of Traditional Chinese Medicine (TCM), comprehensive traditional Chinese therapy is often used to treat Granulomatous Lobular Mastitis (GLM), but the effectiveness and risk are still controversial. This study is aimed to evaluate the efficacy of comprehensive therapy of traditional Chinese medicine on GLM.
\end{abstract}

Methods: Articles in both international databases (PubMed, EMBASE, Cochrane Library, Web of Science and Clinicaltrials.gov) and Chinese databases (Chinese National Knowledge Internet (CNKI), Chinese Biomedical Databases (CBM), VIP Chinese periodical service platform and Wan Fang Digital Journals)) were searched. Original studies which reported the effective rate and/or recurrence rate and/or maximum diameter of the mass of comprehensive traditional Chinese therapy were included. The $95 \%$ confidence interval $(95 \% \mathrm{CI})$ for effective rate, recurrence rate and maximum diameter of the mass were calculated and analyzed by review manager 5.3.

Results: Eight eligible trials with 309 cases and 265 controls were included, six in Chinese and two in English. Statistical analysis suggested a statistical difference in effective rate $(R R=0.86$, $95 \%$ CI $[0.74,1.00], P=0.047)$ between comprehensive traditional Chinese therapy group and control. Meanwhile, there was statistical difference found in recurrence rate between comprehensive traditional Chinese therapy and western medicine therapy $(R R=3.09,95 \% C I$ [1.50, 6.40], $P=0.002$ ). Besides, no statistical difference existed in maximum diameter of the mass between the two therapies $(R R=-5.25,95 \%$ CI [-125.42, 114.93], $P=0.93)$.

Conclusion: Although there was no significant difference in the reduction of breast mass size between comprehensive traditional Chinese therapy and western medicine alone, comprehensive traditional Chinese therapy demonstrated the efficacy in improving the effective rate and reducing the recurrence rate. For GLM patients, comprehensive traditional Chinese therapy could be a potential option.

Keywords: Granulomatous lobular mastitis, Comprehensive traditional Chinese therapy, Systematic review, Meta-analysis, Effectiveness, Recurrence, Maximum diameter of the mass

\section{Introduction}

Granulomatous lobular mastitis (GLM) is an Idiopathic Granulomatous Mastitis (IGM) that 
mainly encroaches on the breast lobule. And it is a rare benign inflammatory breast disease to which women of childbearing age are susceptible ${ }^{[1][2]}$. It is often considered as an autoimmune disease which was first proposed by Kessler et $\mathrm{al}^{[3]}$ in 1972. Because there are many other causes of GLM, such as malignant tumors, infectious diseases and hormone disorders ${ }^{[4]}$, appropriate diagnosis methods are needed to be used.

At present, although no standardized treatment principle has been established in clinical treatment for GLM due to the complicated etiologies ${ }^{[5]}$, surgical treatment and conservative treatment are commonly used against $\mathrm{GLM}^{[6][7]}$. Surgical treatment is possible to leave extensive breast scarring and affect the beauty of the breast ${ }^{[8]}$. Corticosteroid therapy, which is a common conservative treatment, has a high failure rate and many possible side effects ${ }^{[9]}$. Thus, to find an effective treatment is in line with the clinical needs.

Traditional Chinese medicine (TCM), as a conservative treatment, is often used to treat breast diseases ${ }^{[10]}$, but there is still a lack of systematic evaluation on the clinical effectiveness and risk of comprehensive traditional Chinese therapy on GLM. This study included 6 randomized controlled trials (RCT) of the treatment of GLM with comprehensive traditional Chinese therapy. We concluded a meta-analysis to evaluate and summarize the clinical evidence on comprehensive traditional Chinese therapy for the cure of GLM. Herein, we aimed to provide further evidencebased basis for TCM treatment on GLM, so as to provide new insights on GLM treatment.

\section{Methods}

\section{Selection criteria}

Inclusive criteria.

1. Study design: published research articles, all of them were randomized controlled trials, not including cohort studies and case-control studies. Case reports, experience summaries, review articles and meta-analysis were also excluded.

2. Languages: Chinese or English.

3. Groups: The experimental group was treated by comprehensive traditional Chinese medicine alone or combined with western medicine, while the control group was treated by single western medicine, including surgery and/or corticosteroid therapy.

4. Study indications: the effective rate and/or recurrence rate and/or maximum diameter of the mass.

5. Full text: available.

Exclusive criteria.

1. Neither Chinese nor English.

2. The experimental group and/or control group inconsistent with the research contents.

3. Repeated studies.

4. Outcome indicators without effective rate and/or recurrence rate and/or maximum diameter of the mass in studies about GLM.

\section{Search strategy}

We retrieved a number of electronic databases for original published articles, including PubMed, EMBASE, Cochrane Library, Web of Science, Clinicaltrials.gov, CNKI, CBM, VIP Chinese periodical service platform and Wan Fang Digital Journals. The retrieval time ranged from the establishment of the databases to April, 2020. In the retrieval of all the databases, MESH and free term words were added on the basis of GLM, including traditional Chinese medicine, 
acupuncture and massage. The retrieval strategy from PubMed was shown here.

(traditional Chinese medicine OR Chung I Hsueh OR Hsueh, Chung I OR traditional medicine, Chinese OR Zhong, Yi Xue OR Chinese traditional medicine OR Chinese medicine, traditional $O R$ traditional tongue diagnosis $O R$ tongue diagnoses, traditional OR tongue diagnosis, traditional OR traditional tongue diagnoses OR traditional tongue assessment $O R$ tongue assessment, traditional $O R$ traditional tongue assessments $O R$ acupuncture $O R$ acupuncture therapy $O R$ acupuncture, ear $O R$ acupuncture points $O R$ acupuncture analgesia OR massage OR heart massage OR Transtympanic Micropressure treatment OR manual lymphatic drainage OR musculoskeletal manipulations) AND (granulomatous mastitis)

Clinical studies, review articles, animal experiments and meeting records were clustered manually and classified according to the retrieval results.

Study selection, data extraction, methodological quality and quality of evidence evaluation

Study selection, data extraction, methodological quality and quality of evidence evaluation were carried out by two researchers independently in strict conformity with the inclusive and exclusive criteria. In case of any disagreement, the two researchers discussed and solved it through consultation. The problems and differences unresolved were carried out by a third reviewer.

\section{Data analysis and statistical considerations}

The main purpose of the meta-analysis was to compare the effective rate, the recurrence rate and the maximum diameter of the mass in two different treatment groups. Calculating and adding up all the outcome data of each indications was the first step to make the study accurate. The second step was that all analyses were stratified by experiments. Then Review Manager 5.3 was used to conduct statistical analysis on the collected data. For the effectiveness analysis of research indicators, in order to discuss the differences between different intervention methods, subgroup data analysis was undertaken. Effects on dichotomous data were summarized by risk ratio $(R R)$ or odds ratio $(O R)$, and influences on continuous data were summarized with weighted mean difference $(W M D)$ or standardized mean difference $(S M D)$ to measure the risk of appearing certain outcomes in the experimental group as compared to the control group. The $95 \%$ confidence interval $(C I)$ was shown by a forest plot in RevMan5.3. The heterogeneity test was responded by P-value and $I^{2}$. If P-value was more than 0.05 and $I^{2}$ was less than 50\%, it implied there was no statistical significance in heterogeneity. On the contrary, it implied there was significance in heterogeneity if P-value was less than 0.05 or $I^{2}$ was more than $50 \%$.

\section{Results}

Trials and patients description

We retrieved 219 relevant literatures, of which 8 were finally included in our analysis. The studies selection process was shown in Figure 1. All the experimental studies included were randomized controlled trials. Effective rate was not reported in two studies, recurrence rate was reported in three studies but maximum diameter of the mass was only reported in two studies. All the patients were diagnosed with GLM. There were 265 cases in control group and 309 cases in experimental group. The information included in the literature was shown in Table 1. 


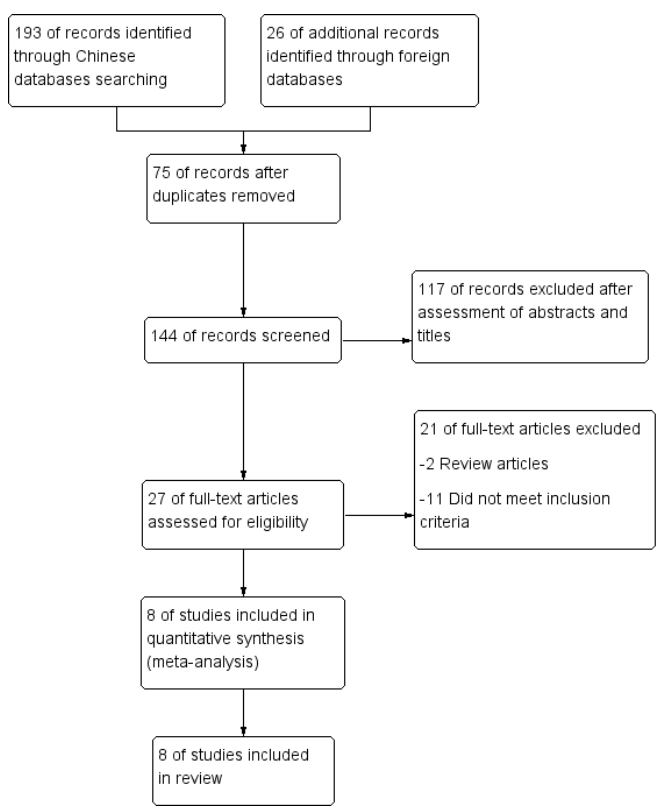

Figure 1. Flowchart of the studies selection

Table 1. Basic information of included studies

\begin{tabular}{|c|c|c|c|c|c|c|c|c|}
\hline First author & $\begin{array}{c}\text { Published } \\
\text { Year }\end{array}$ & $\begin{array}{c}\text { N. of } \\
\text { C }\end{array}$ & $\begin{array}{c}\text { N. of } \\
\text { E }\end{array}$ & Age in $\mathrm{C}$ & Age in $\mathrm{E}$ & $\begin{array}{l}\text { Intervention } \\
\text { in } \mathrm{C}\end{array}$ & $\begin{array}{l}\text { Intervention } \\
\text { in } \mathrm{E}\end{array}$ & $\begin{array}{c}\text { Follow-up } \\
\text { Time }\end{array}$ \\
\hline Zhao HD ${ }^{[11]}$ & 2019 & 91 & 91 & $31.24 \pm 4.21$ & $31.59 \pm 4.13$ & 1(a) & $3(\mathrm{c} \mathrm{e})$ & NA \\
\hline Guo $Z^{[12]}$ & 2016 & 24 & 24 & NA & NA & $1(a b)$ & $2(\mathrm{acd})$ & 6-8months \\
\hline Yang $L^{[13]}$ & 2017 & 20 & 40 & $30.6 \pm 5.1$ & $31.3 \pm 5.6$ & 1(a) & $3(\mathrm{c} \mathrm{d} \mathrm{f})$ & NA \\
\hline Zheng $\mathrm{MN}^{[14]}$ & 2018 & 20 & 20 & $30.40 \pm 5.70$ & $30.95 \pm 4.82$ & 1(a) & $3(\mathrm{c} \mathrm{d})$ & NA \\
\hline Liu HR ${ }^{[15]}$ & 2017 & 24 & 38 & $30.12 \pm 3.53$ & $31.26 \pm 2.35$ & $1(\mathrm{a} \mathrm{b})$ & $2(a b c d)$ & NA \\
\hline Xiao $\mathrm{JZ}^{[16]}$ & 2018 & 16 & 16 & $40.0 \pm 6.6$ & $36.1 \pm 5.1$ & 1(a) & $2(\mathrm{a} \mathrm{c} \mathrm{d})$ & NA \\
\hline Xue JX $X^{[17]}$ & 2020 & 20 & 20 & $34.65 \pm 7.49$ & $34.35 \pm 4.42$ & 1(a) & $3(\mathrm{~d})$ & 4weeks \\
\hline Liu $P Z^{[18]}$ & 2020 & 50 & 60 & $30.72 \pm 2.701$ & $30.40 \pm 3.43$ & $1(\mathrm{a} b)$ & $2(\mathrm{a} b \mathrm{~cd})$ & 1year \\
\hline
\end{tabular}

Note: Interventions, "1" means simple western medicine treatment, "2" means combination of

Chinese and western medicine treatment, " 3 " means comprehensive traditional Chinese therapy.

"a" means corticosteroid therapy, "b" means surgical treatment, "c" means herbal oral

administration, "d" means external application Chinese medicine, "e" means massage, "f" means

acupuncture treatment.

"C" means control group.

"E" means experimental group.

"NA" means not available.

Quality evaluation

8 studies were included and Cochrane quality evaluation form was used to assess the bias risk. High risk of bias was represented by red, unclear risk of bias was represented by yellow and low risk of bias was represented by green. Risk of bias graph was about each risk of bias item presented as percentages across all included studies in our judgements. Besides, risk of bias summary was about each risk of bias item for each included study. The details were shown in the following Figure 2 and 3. 


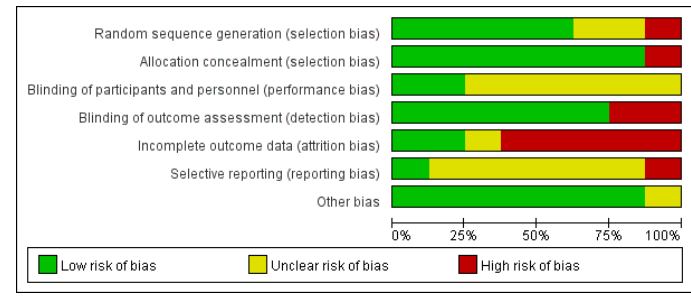

Figure 2. Risk of bias graph

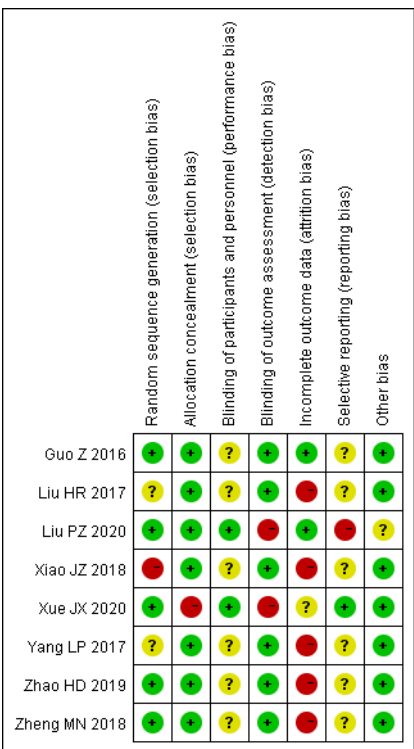

Figure 3. Risk of bias summary

Effective rate of GLM patients

In all the studies, 6 studies recorded effective rate of the treatments. Effective rate study was a dichotomous data study, so $R R$ was used for analysis. The heterogeneity among the studies was high $\left(P=0.0005<0.05, I^{2}=78 \%>50 \%\right)$. Besides, there was a statistical difference between comprehensive traditional Chinese therapy of GLM and western medicine therapy $(R R=0.86$, $95 \%$ CI $[0.74,1.00], P=0.047<0.05)$ (Figure 4).

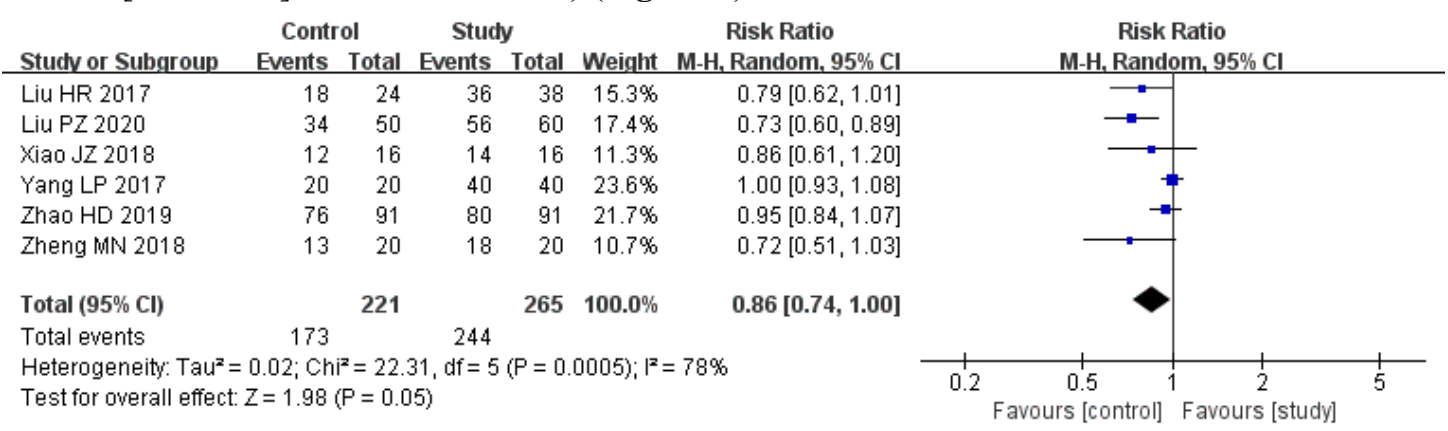

Figure 4. Forest plot of the comparison of effectiveness between comprehensive traditional Chinese therapy and western medicine therapy (The result from Review Manager 5.3-study comparison)

Recurrence rate of GLM patients

Among all the studies, only 3 studies stated recurrence rate of the treatments. Recurrence rate study was also a dichotomous data study, so $R R$ was used for analysis. There was no heterogeneity in the study $\left(P=0.97>0.05, I^{2}=0 \%<50 \%\right)$. In addition, there was a significant difference between comprehensive traditional Chinese therapy of GLM and western medicine therapy $(R R=$ $3.09,95 \%$ CI [1.50,6.40], $P=0.002<0.05$ ) (Figure 5). 


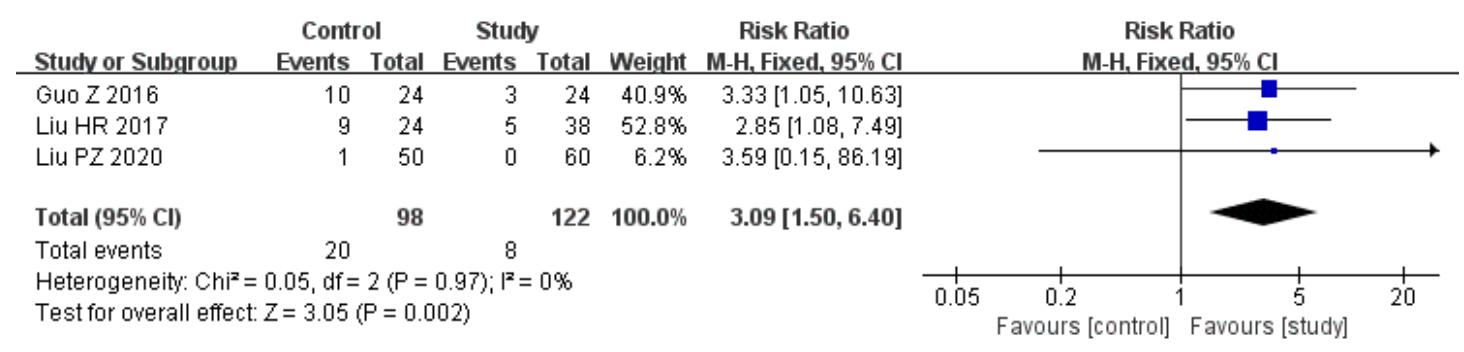

Figure 5. Forest plot of the comparison of recurrence between comprehensive traditional Chinese therapy and western medicine therapy (The result from Review Manager 5.3-study comparison)

Maximum diameter of the mass in GLM patients

Among all the studies, maximum diameter of the mass was indicated in 2 studies. Size of the mass and maximum diameter of the focus were all used to represent maximum diameter of the mass. Maximum diameter of the mass study was a continuous data study, so WMD was used for analysis. There was only homogeneity but no heterogeneity in the studies $\left(P=0.98>0.05, I^{2}=\right.$ $0 \%<50 \%$ ). Besides, there was no statistical difference between comprehensive traditional Chinese therapy of GLM and western medicine therapy ( WMD $=-5.25,95 \%$ CI [-125.42, 114.93], $P=0.93>0.05$ ) (Figure 6).

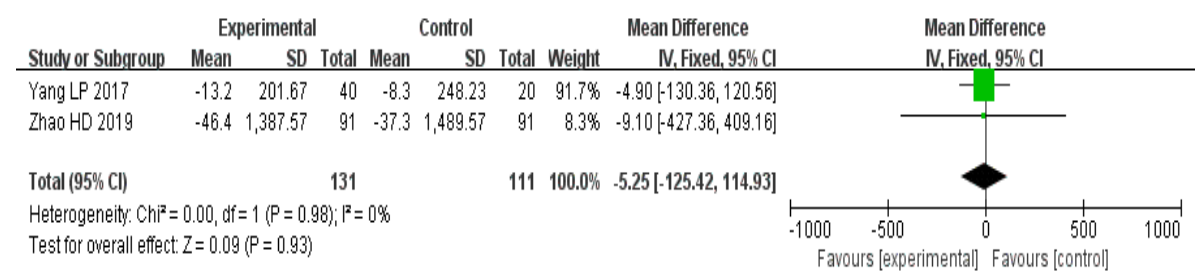

Figure 6. Forest plot of the comparison of maximum diameter of the mass between comprehensive traditional Chinese therapy and western medicine therapy (The result from Review Manager 5.3study comparison)

\section{Discussion}

We made a comprehensive analysis of the effective rate, recurrence rate and maximum diameter of the mass of comprehensive traditional Chinese therapy in the treatment of GLM in this study. The effective rate study included 486 patients, in which the effective rate was $78.3 \%$ in control group and that was $92.1 \%$ in experimental group. Besides, the recurrence rate included 110 patients, in which the recurrence rate was $20.4 \%$ in control group and that was $6.56 \%$ in experimental group. In addition, 242 patients were included in the maximum diameter of the mass study. Effectiveness and safety were judged by effective rate and recurrence rate ${ }^{[19]}$. The comprehensive estimation of the effective rate and the recurrence rate was called the curative effect of comprehensive traditional Chinese therapy ${ }^{[20]}$. And the recovery was reflected by the change of maximum diameter to some extent. This study suggested that not only there was a significant effect on the increase of the effective rate in treating GLM, but also the effective rate in experimental group was higher than in control group. And the extent of mass reduction in experimental group was also better than in control group. Thus, in clinical analysis, the effectiveness was improved, the recurrence rate was reduced and the size of breast mass was decreased by comprehensive traditional Chinese therapy. We summarized that the treatment of 
GLM by comprehensive traditional Chinese therapy was potential to be advocated in clinical use.

In recent years, the significant effect of traditional Chinese medicine treatment on GLM had been reported by many clinical studies and investigations. For instance, in traditional Chinese herbal medicine, CCL-5 in Broadleaf Mahonia reported by Wang $\mathrm{Z}$ et a ${ }^{[21]}$ was related to the pathogenesis of GLM and the prognosis of GLM was effectively improved by this safe and effective natural Chinese medicine. Taraxaci Herba, Glycyrrhizae Radix et Rhizoma, Paeoniae Radix Alba and semen citri reticulatae explored by Wu D et a ${ }^{[22]}$ were used as effective drugs in combination of Chinese materia medica to treat mastitis through data mining and results researching. According to the analysis of pharmaceutical components and summary of main clinical application, Suo S et al ${ }^{[23]}$ concluded that Amomi fructus was also effective in the treatment of mastitis. Rose, as an herb, was used to treat mastitis, which was recorded in Cheng L's article ${ }^{[24]}$. The active role of Solanum nigrum Unripe fruit fraction in the treatment of mastitis was also known by Jagadeeshan, $\mathrm{S}$ et $\mathrm{al}^{[25]}$ on studying it. The Catpesium plants, recorded by Zhang J, were used in many countries, and outstanding effect in the treatment of mastitis was found in its hemostatic, anti-inflammatory and detoxifying effect ${ }^{[26]}$. Lonicera japonica Thunb, as a widely used traditional Chinese herbal medicine, had a prominent therapeutic effect on breast abscess $^{[27]}$. The herbs mentioned above summarized the commonly application in the treatment of mastitis. In this study, six studies were all treated with orally traditional Chinese herbal medicine, and all of the traditional Chinese herbal medicine were combined into prescriptions. Xiao Zhong powder, Xiao Yao Lou Bei powder, Si Zi powder, Tou Nong powder, San Jie Xiao Zhong decoction, Huang Lian Wen Dan decoction, Yang He decoction and Tiao Qi He Ying decoction were all combination of Chinese herbal medicine. These prescriptions were used to eliminate swelling and pain at the initial stage. At the middle stage, they were used to activate blood circulation and remove stasis, and at the later stage, they were used to strengthen the body and remove blood stasis. The prescriptions were easy to fry and boil. Based on researching the effective statistical analysis, clinical analysis of recurrence rate and recovery of mass size, the therapeutic effect was effectively improved by traditional Chinese herbs medicine.

In addition, the application of traditional Chinese medicine in GLM was not limited to the application of traditional Chinese herbal medicine. As Ma X et a ${ }^{[28]}$ said, the observation was an important choice for early treatment of GLM. Similarly, catheter lavage and breast massage which were considered as conservative treatment methods had little harm and little side effects ${ }^{[29]}$. And they were their unique advantages. The manipulation of kneading and dispersing was similar to that of breast massage, which had its own characteristic in reducing breast mass and improving clinical symptoms ${ }^{[30]}$. Cytological study showed that not all of granulomatous mastitis was tuberculosis, which also emphasized the conservative treatment ${ }^{[31]}$. A variety of traditional Chinese medicine methods were combined in our study. 1 literature used massage, 1 literature used acupuncture therapy and 5 literatures used external treatment of traditional Chinese medicine except herbal oral administration. Through the comparison of these comprehensive therapies with surgical treatment and corticosteroid therapy, we found that the reduction of recurrence rate was better in comprehensive traditional Chinese therapy than single western therapy. In a word, the combination of traditional Chinese medicine treatment was worthy of our clinical promotion.

According to this meta-analysis, there was a significant difference in the effective rate and recurrence rate of the treatment of GLM with comprehensive traditional Chinese therapy. Besides, the effective rate was significantly increased and the recurrence rate was significantly decreased. 
However, the maximum diameter of the mass was not statistically increased or decreased by comprehensive traditional Chinese therapy compared with single western therapy. However, from a clinical point of view, we could not conclude that there was no difference between comprehensive traditional Chinese therapy and simple western medicine for GLM. Therefore, more standard large sample clinical observation should be designed to evaluate the efficacy of comprehensive traditional Chinese therapy on GLM. There were still some limitations in our study: 1. Most of the literature we included were from China, and there was little international researches that met the inclusion conditions. Uneven distribution of samples in country, nationality and other aspects were resulted in this situation, and not convincing research results were gotten. Just as our study on the effective rate analysis, we learned that a source of heterogeneity was existed in the comparison between single western medicine treatment and comprehensive traditional Chinese therapy and the comparison between single western medicine treatment and comprehensive traditional Chinese therapy combined with western medicine. However, further analysis was not made, so more researches on heterogeneity were still needed. 2 . The sample size we included was not large enough, and the patients had differences in age, region and other aspects. And although all the studies we included were randomized controlled trials, the quality of the literature included was low according to the scores of Cochrane manual. As shown in Figure 2 and Figure 3, the blinding of participants and personnel and selective reporting of all the studies were unknown, and high risk of bias about incomplete result data was obtained in most of the studies. The dispersion and shape of the forest plot were affected by all of the difference and bias. In short, the pooled estimates of effective rate, recurrence rate and maximum diameter of the mass were influenced by them. However, our studies included were all in recent years. In the existing literature on the treatment of GLM by traditional Chinese medicine, this systematic review and meta-analysis of randomized controlled trials was still of referential value.

\section{Conclusions}

This meta-analysis of GLM therapies indicated that: (1) the effective rate in comprehensive traditional Chinese therapy was significantly different from western medicine therapy, and clinically improved. (2) comprehensive therapy of traditional Chinese medicine had a lower recurrence rate in treating GLM compared with single western medicine treatment, and no heterogeneity existed. (3) the maximum diameter of the mass in comprehensive traditional Chinese therapy was not statistically different from simple western medicine therapy, but breast mass was reduced clinically.

\section{Ethics approval and consent to participate}

The systematic review and meta-analysis were approved by the Ethics Committee of Shandong University of Traditional Chinese Medicine.

\section{Consent for publication}

All authors agree the publishment.

\section{Availability of data and materials}

Please contact authors for data requests. 


\section{Competing interests}

All authors declare that there are no conflict of interests.

\section{Funding and Acknowledgements}

This study was supported by the National Natural Science Foundation of China (No.81573989 and No.81403408), and Traditional Chinese medicine science and technology development plan project in Shandong Province (No.2019-0159).

\section{Authors' contributions}

H.L., H.C., G.S., X.L., and J.L. collected and analyzed the data. H.L. wrote the manuscript. J.L. checked up the manuscript. All authors reviewed and approved the final manuscript. 


\section{References}

[1] Freeman CM, Lewis JD, Lower EE, et al. Refractory granulomas of breast: Benign or malignant disease. Journal of Clinical Oncology, 2014; 32(26).

[2] Goulabchand R, Hafidi A, Van de Perre P, et al. Mastitis in Autoimmune Diseases: Review of the Literature, Diagnostic Pathway, and Pathophysiological Key Players. J Clin Med, 2020; 9(4).

[3] Kessler E, Wolloeh Y. Granulomatous mastiffs: a lesion clinically simulating carcinoma. Am J Clin Pathol, 1972; 58(6): 642-6.

[4] Lei X, Chen K, Zhu L, et al. Treatments for Idiopathic Granulomatous Mastitis: Systematic Review and Meta-Analysis. Breastfeed Med, 2017; 12(7): 415-21.

[5] Freeman CM, Xia BT, Wilson GC, et al. Idiopathic granulomatous mastitis: A diagnostic and therapeutic challenge. Am J Surg, 2017; 214(4): 701-6.

[6] Granat A, Brizuela M, Macdonald H, et al. The treatment conundrum for idiopathic granulomatous mastitis. Annals of Surgical Oncology, 2017; 24(2): 66-7.

[7] Zhou F, Liu L, Liu L, et al. Comparison of Conservative versus Surgical Treatment Protocols in Treating Idiopathic Granulomatous Mastitis: A Meta-Analysis. Breast Care, 2019.

[8] Shin YD, Park SS, Song YJ, et al. Is surgical excision necessary for the treatment of Granulomatous lobular mastitis? BMC Womens Health, 2017; 17(1): 49.

[9] Yukawa M, Watatani M, Isono S, et al. Management of granulomatous mastitis: a series of 13 patients who were evaluated for treatment without corticosteroids. Int Surg, 2015; 100(5): 774-82. [10] Chung VC, Wu X, Hui EP, et al. Effectiveness of Chinese herbal medicine for cancer palliative care: overview of systematic reviews with meta-analyses. Vol. 5. 2015. 18111.

[11] Hui-duo Z, Ai-jing C, Wei W, et al. A study on the curative effect of xiaozhongsan combined with massage on granulomatous mastitis. Shaanxi Journal of Traditional Chinese Medicine, 2019; 40: 743-5.

[12] Zhen G, Li X. A study on the Comprehensive Therapy and Plastic Surgical Repair of Granulomatous Mastitis. Journal of Jiangxi University of TCM, 2016; 28: 37-40.

[13] Le-ping Y, Yun H, Wei-zuo L, et al. Comparison of ultrasound and clinical effect of external treatment of traditional Chinese medicine and hormone block treatment of granulomatous mastitis. Modern Journal of Integrated Traditional Chinese and Western Medicine, 2017; 26: 2546-8.

[14] Mei-na Z. Observation on the curative effect of sanjiexiaozhong decoction combined with sanhuang plaster in the treatment of granulomatous mastitis. Chin J School Doctor, 2018; 32

[15] He-rong L. Observation on the curative effect of treating granulomatous lobular mastitis with the combination of traditional Chinese and western medicine. Nei Mongol Journal of Traditional Chinese Medicine, 2017; 6: 68-9.

[16] Jia-zhe X, Ming-qin L, Li-jun L. Randomized parelled controlled clinical study on tiaoqi heying method combined with corticosteroids in the treatment of granulomatous mastitis. CHINA MODERN DOCTOR, 2018; 56: 127-9.

[17] Jing-xian Xue, Bei Ye, Shun Liu, et al. Treatment Efficacy of Chuang Ling Ye, a Traditional Chinese Herbal Medicine Compound, on Idiopathic Granulomatous Mastitis: A Randomized Controlled Trial, Evid Based Complement Alternat Med, 2020; 2020: 6964801.

[18] Liu PZ, Shi XG, Zuo XM, et al. A clinical study on the treatment of granulomatous lobular mastitis by the external application of the internal pus-expelling decoction and operation. Ann Palliat Med 2020;9(5):2631-2641.

[19] Pinto M, Bueno L Carrera, D Quispe A, Effectiveness and safety attributable to oncoplastic surgery as an adjuvant therapy for idiopathic granulomatous mastitis due to breast tuberculosis. Annals of Surgical Oncology, 2019. 26(2): 131-132.

[20] Chen L, Zhang XY, Wang YW, et al. Granulomatous lobular mastitis: a clinicopathological analysis of 300 cases. Zhonghua Bing Li Xue Za Zhi, 2019. 48(3): 231-6.

[21] Wang Z, Wang N, Liu X, et al. Broadleaf Mahonia attenuates granulomatous lobular mastitis-associated inflammation by inhibiting CCL-5 expression in macrophages. Int J Mol Med, 2018. 41(1): 340-52.

[22] Wu D, Zhang X, Liu L, et al. Key CMM Combinations in Prescriptions for Treating Mastitis and Working Mechanism Analysis Based on Network Pharmacology. Evid Based Complement Alternat Med, 2019. 8245071.

[23] Suo S, Lai Y, Li, M, et al. Phytochemicals, pharmacology, clinical application, patents, and products of Amomi fructus. Food Chem Toxicol, 2018; 119: 31-6. 
[24] Cheng L, Y Zhen. Comparison on the function of rose as interpreted by traditional Chinese medicine and medicine of Uighur ethnic group. Zhonghua Yi Shi Za Zhi, 2017; 47(6): 366-8.

[25] Jagadeeshan S, David D, Jisha S, et al. Solanum nigrum Unripe fruit fraction attenuates Adriamycin resistance by down-regulating multi-drug resistance protein (Mdr)-1 through JakSTAT pathway. BMC Complement Altern Med, 2017; 17(1): 370.

[26] Zhang J, Wang Guo-Wei, Tian Xin-Hui, et al. The genus Carpesium: A review of its ethnopharmacology, phytochemistry and pharmacology. JOURNAL OF

ETHNOPHARMACOLOGY, 2015; 163: 173-191.

[27] Shang X, Pan H, Li M, et al. Ethnopharmacology, phytochemistry and pharmacology of an important traditional Chinese medicine. Journal of Ethnopharmacology, 2011; 138(1): 1-21.

[28] Ma X, X Min and C Yao. Different Treatments for Granulomatous Lobular Mastitis: A Systematic Review and Meta-Analysis. Breast Care (Basel), 2020; 15(1): 60-6.

[29] Li S, Chen K, Zhu L, et al. Efficacy and safety of ductal lavage for non-lactational idiopathic granulomatous mastitis: A retrospective case series study. Annals of Surgical Oncology, 2017;

24(2): 71-2.

[30] Zhou M, Li X, Cheng YQ, et al. Kneading and dispersing manipulation in treatment of earlystage acute mastitis: a randomized controlled trial. Zhong Xi Yi Jie He Xue Bao, 2009; 7(12):

1130-3.

[31] Agarwal C, Singh K, Pujani M, et al. Are all Granulomatous Mastitis Cases Tuberculous ? : A Study on the Role of Cytology in Evaluation of Granulomatous Mastitis. Turk Patoloji Derg, 2019; 35(2): 128-33. 
Figures

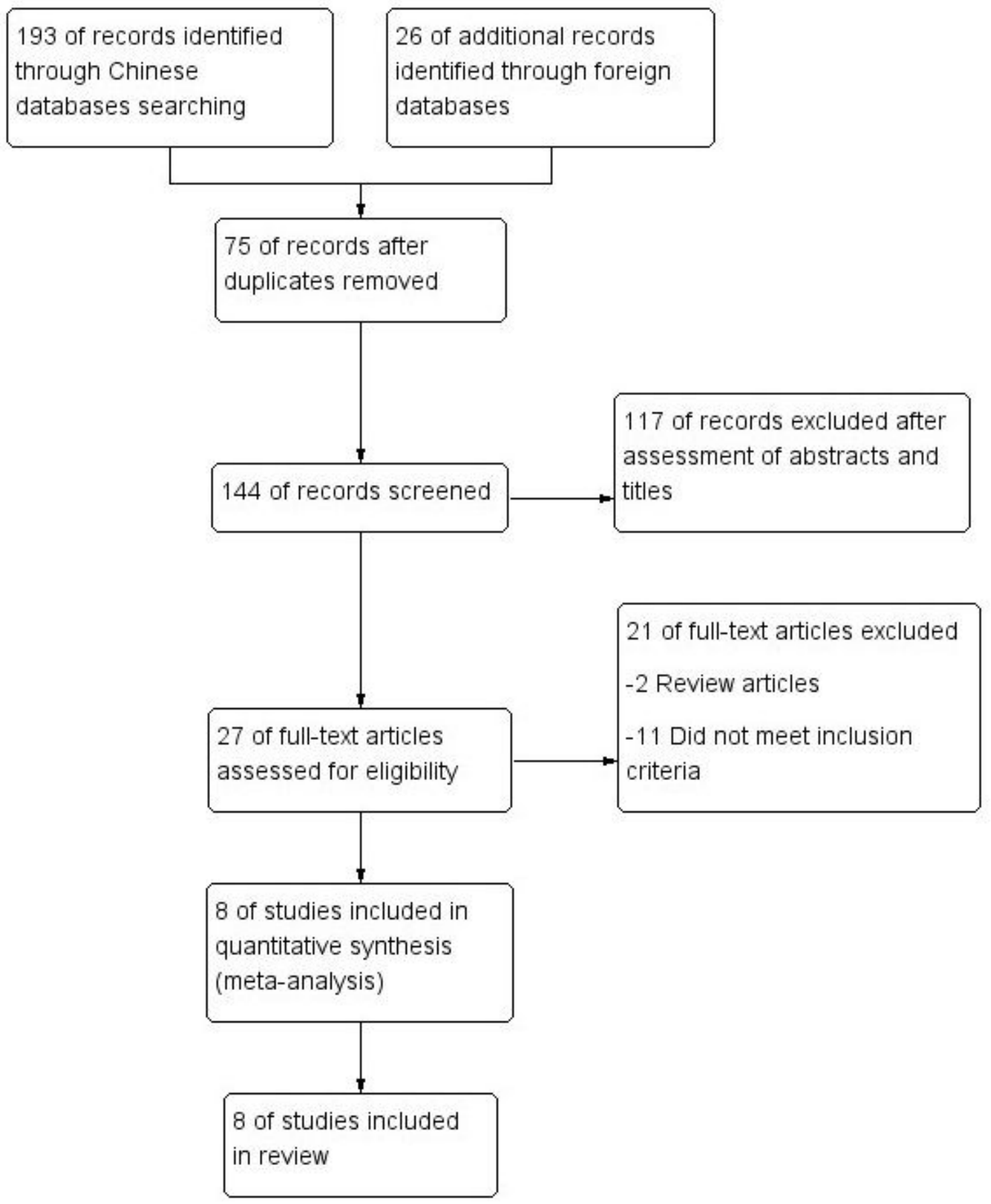

Figure 1

Flowchart of the studies selection 


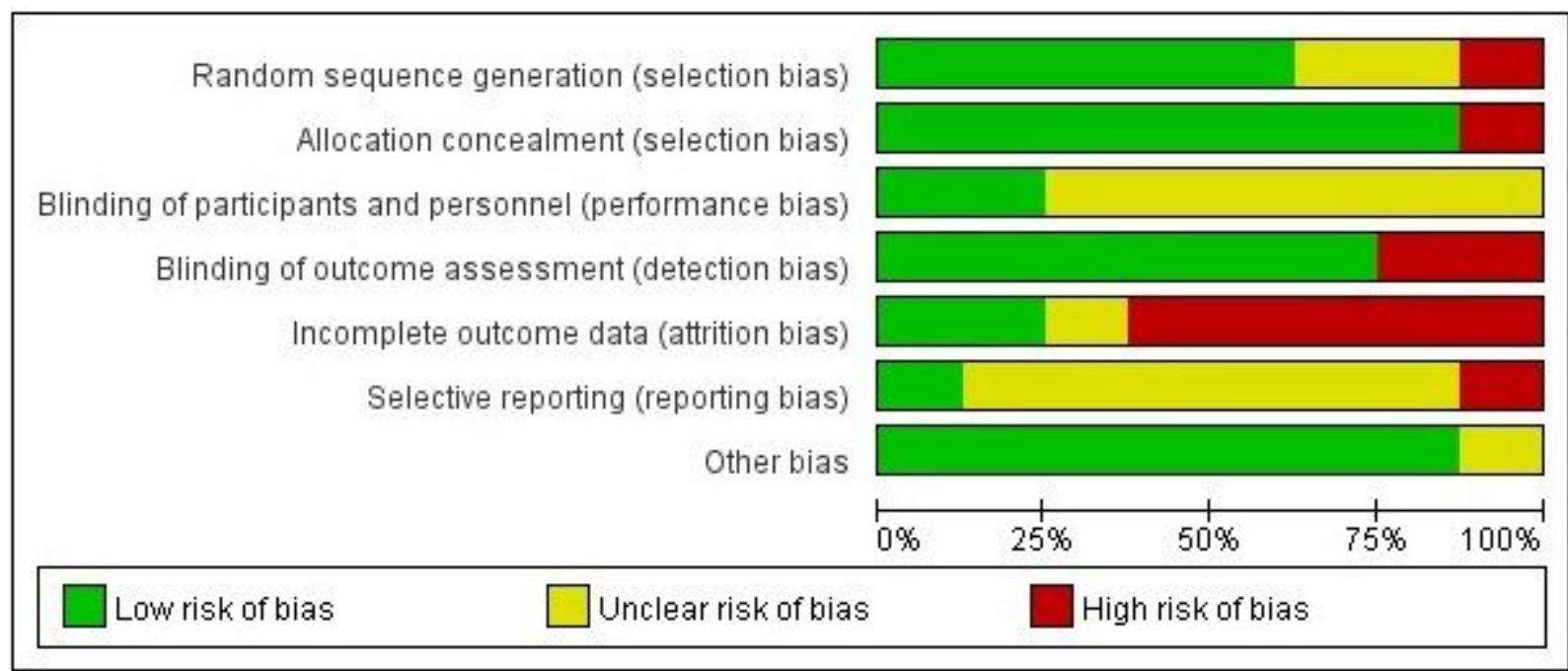

Figure 2

Risk of bias graph 


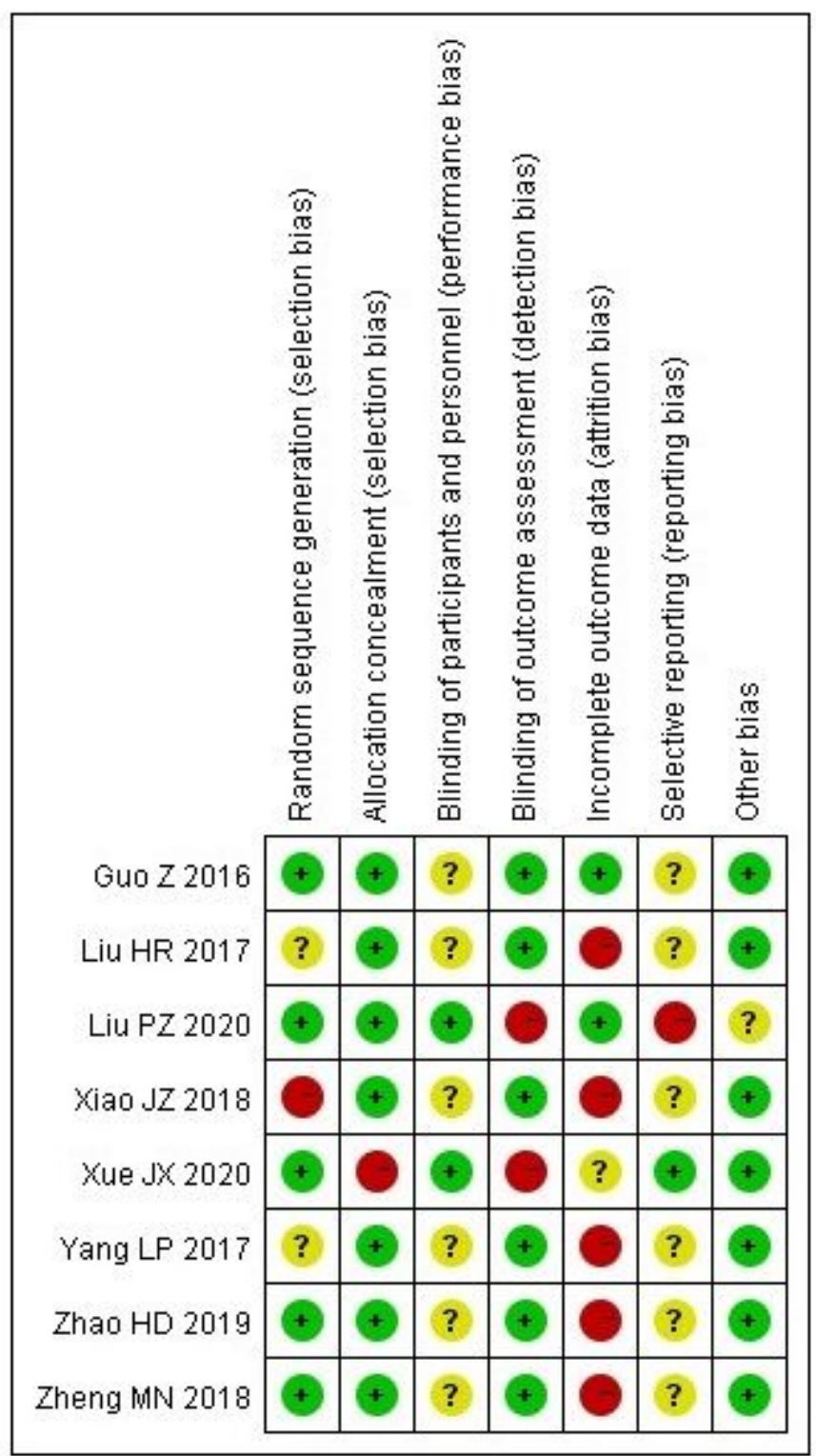

\section{Figure 3}

Risk of bias summary

Control

Study

Risk Ratio

Risk Ratio

Studv or Subgroup Events Total Events Total Weight M-H, Random, 95\% Cl

Liu HR 2017

Liu PZ 2020

Xiao JZ 2018

Yang LP 2017

Zhao HD 2019

Zheng MN 2018

Total $(95 \% \mathrm{CI})$

Total events

Heterogeneity: $\operatorname{Tau}^{2}=0.02 ; \mathrm{Chi}^{2}=22.31, \mathrm{df}=5(\mathrm{P}=0.0005) ; \mathrm{I}^{2}=78 \%$

Test for overall effect: $Z=1.98(P=0.05)$
$0.79[0.62,1.01]$

$0.73[0.60,0.89]$

$0.86[0.61,1.20]$

$1.00[0.93,1.08]$

$0.95[0.84,1.07]$

$0.72[0.51,1.03]$

$0.86[0.74,1.00]$

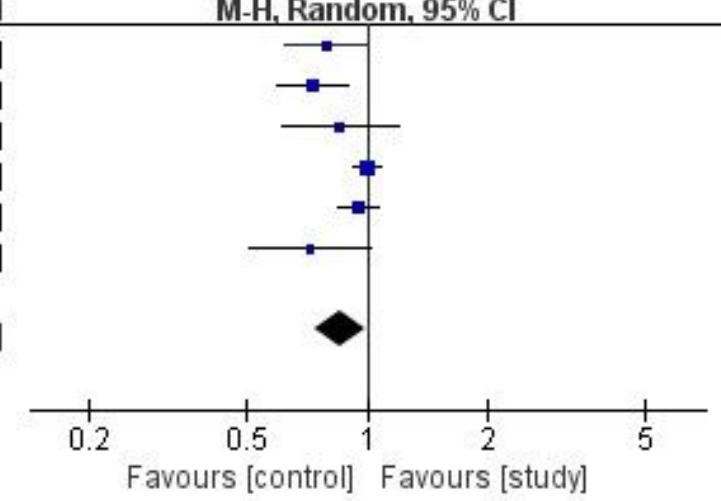


Forest plot of the comparison of effectiveness between comprehensive traditional Chinese therapy and western medicine therapy (The result from Review Manager 5.3-study comparison)

\begin{tabular}{|c|c|c|c|c|c|c|c|c|c|c|}
\hline \multirow{2}{*}{ Studv or Subgroup } & \multirow{2}{*}{\multicolumn{2}{|c|}{$\begin{array}{l}\text { Control } \\
\text { Events }\end{array}$}} & \multicolumn{2}{|c|}{ Study } & \multirow{2}{*}{\multicolumn{2}{|c|}{$\begin{array}{cc}\text { Risk Ratio } \\
\text { Weight } & \text { M-H, Fixed, } 95 \% \mathrm{Cl} \\
\end{array}$}} & \multirow{2}{*}{\multicolumn{4}{|c|}{$\begin{array}{l}\text { Risk Ratio } \\
\text { M-H. Fixed } 95 \%\end{array}$}} \\
\hline & & & vents & Total & & & & & & \\
\hline Guo Z 2016 & 10 & 24 & 3 & 24 & $40.9 \%$ & $3.33[1.05,10.63]$ & & & प? & \\
\hline Liu HR 2017 & 9 & 24 & 5 & 38 & $52.8 \%$ & $2.85[1.08,7.49]$ & & & & \\
\hline Liu PZ 2020 & 1 & 50 & 0 & 60 & $6.2 \%$ & $3.59[0.15,86.19]$ & & & & \\
\hline Total (95\% Cl) & & 98 & & 122 & $100.0 \%$ & $3.09[1.50,6.40]$ & & & & \\
\hline Total events & 20 & & 8 & & & & & & & \\
\hline $\begin{array}{l}\text { Heterogeneity: } \mathrm{Chi}^{2}= \\
\text { Test for overall effect }\end{array}$ & $\begin{array}{l}0.05, d f= \\
Z=3.05(t\end{array}$ & $\begin{array}{l}(P=0 \\
=0.00\end{array}$ & & & & & 0.05 & $\begin{array}{ll}0.2 \\
\text { vours [controll] }\end{array}$ & $\begin{array}{l}5 \\
\text { [study] }\end{array}$ & 20 \\
\hline
\end{tabular}

Figure 5

Forest plot of the comparison of recurrence between comprehensive traditional Chinese therapy and western medicine therapy (The result from Review Manager 5.3-study comparison)

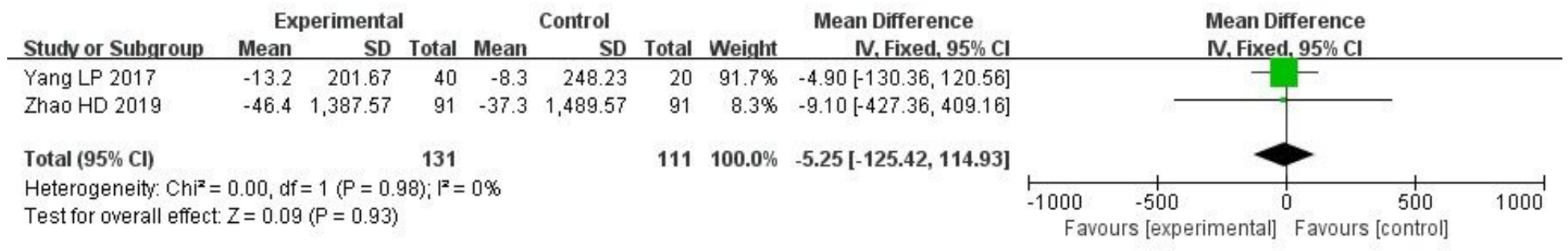

\section{Figure 6}

Forest plot of the comparison of maximum diameter of the mass between comprehensive traditional Chinese therapy and western medicine therapy (The result from Review Manager 5.3-study comparison) 